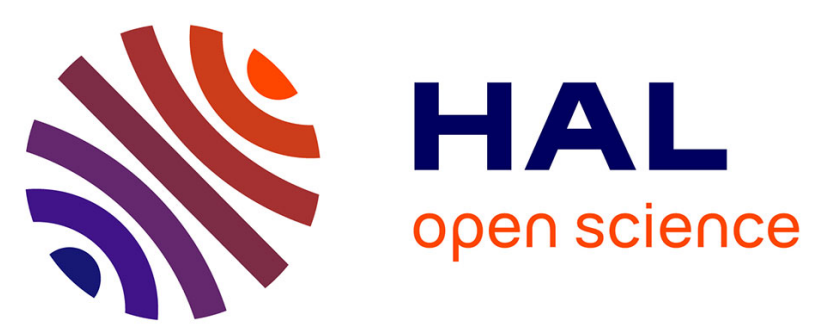

\title{
Effects of a Weak Magnetic Field on Carrier Transport in a Cryogenic Germanium Detector for Dark Matter Search
}

\author{
A. Broniatowski, L. Sengelé, M. Chapellier
}

\section{- To cite this version:}

A. Broniatowski, L. Sengelé, M. Chapellier. Effects of a Weak Magnetic Field on Carrier Transport in a Cryogenic Germanium Detector for Dark Matter Search. 14th International Workshop on Low Temperature Detectors, Aug 2011, Heidelberg, Germany. pp.1143-1148, 10.1007/s10909-012-0546-2 . in2p3-00674808

\author{
HAL Id: in2p3-00674808 \\ https://hal.in2p3.fr/in2p3-00674808
}

Submitted on 28 Feb 2012

HAL is a multi-disciplinary open access archive for the deposit and dissemination of scientific research documents, whether they are published or not. The documents may come from teaching and research institutions in France or abroad, or from public or private research centers.
L'archive ouverte pluridisciplinaire HAL, est destinée au dépôt et à la diffusion de documents scientifiques de niveau recherche, publiés ou non, émanant des établissements d'enseignement et de recherche français ou étrangers, des laboratoires publics ou privés. 
Proceedings $14^{\text {th }}$ International Workshop on Low Temperature Detectors (Heidelberg, Germany, 2011), to appear in Journal of Low Temperature Physics (2012). DOI: 10.1007/s10909-012-0546-2

\title{
Effects of a Weak Magnetic Field on Carrier Transport in a Cryogenic Germanium Detector for Dark Matter Search
}

\author{
A. Broniatowski ${ }^{(*)}$, L. Sengelé and M. Chapellier \\ Centre de Spectrométrie Nucléaire et de Spectrométrie de Masse, \\ IN2P3/CNRS and Université Paris XI, Bât. 108, 91405 Orsay (France)
}

A magnetic field of a few gauss produces sizeable effects on carrier transport and charge collection in a germanium dark matter detector operated at millikelvin temperatures. The magnitude of the effects is explained by the large values of the velocities imparted to the carriers, even under the low electric field conditions typical for charge collection in these devices (a few $10^{6} \mathrm{~cm} / \mathrm{s}$ at $\sim 1 \mathrm{~V} / \mathrm{cm}$ ). Using a suitable experimental setup, effects of the magnetic field on electron and hole transport were investigated separately. A dependence of these effects on the orientation of the field relative to the detector axis is demonstrated, arising in part from magnetic flux conservation through the superconducting (Al) annular electrodes on these devices.

\section{INTRODUCTION}

Cryogenic germanium detectors owe their status in dark matter search experiments to their excellent background rejection capabilities. It is therefore a matter of interest to investigate to what extent carrier transport and charge collection in these devices is affected by factors, whether materialdependent (e.g., variations in the impurity content of the germanium crystals) or external, such as a perturbation by a stray magnetic field. A weak magnetic field can affect electronic transport in high-purity germanium very significantly, given that the carriers acquire high drift velocities even at the low collection fields typical for the operation of the detectors $(<\sim$ one volt per centimeter). For an order of magnitude estimate, we consider the case of holes, whose drift velocity at $\mathrm{mK}$ temperatures under an electric field of 0.5 $\mathrm{V} / \mathrm{cm}$ is about $2 \times 10^{6} \mathrm{~cm} / \mathrm{s} .{ }^{1}$ (Similar values obtain for electrons.) Assuming a magnetic field of 5 gauss ( $\sim$ ten times the terrestrial field) at right angle to the electric field, the ratio of the magnetic to the electric force is $\sim 0.2$,

$\left.{ }^{*}\right)$ corresponding author. e-mail: alexandre.broniatowski@csnsm.in2p3.fr 


\section{A. Broniatowski}

which shows that hole transport should be affected appreciably. We investigate these effects on a dark matter detector at $20 \mathrm{mK}$, using as a source for the magnetic field a permanent (FeNdB) magnet located outside the body of the ${ }^{3} \mathrm{He} /{ }^{4} \mathrm{He}$ dilution refrigerator.

Section 2 presents the setup for these experiments. We compare the effects of the magnetic field on electron and hole transport, which are very different on account of crystal anisotropy (sec. 3). Because the detector is fitted with annular, superconducting aluminum electrodes, supercurrents within the electrodes are expected to shield, at least partly, the core of the detector crystal against variations of a perturbing magnetic field. We discuss in conclusion possible implications of these observations for charge collection in Ge cryogenic detectors.

\section{EXPERIMENTAL SETUP}

The experiment is designed to distinguish between the electron and hole patterns of charge collection in a given set of electric and magnetic field conditions. To this end, advantage is taken of the electrode configuration of

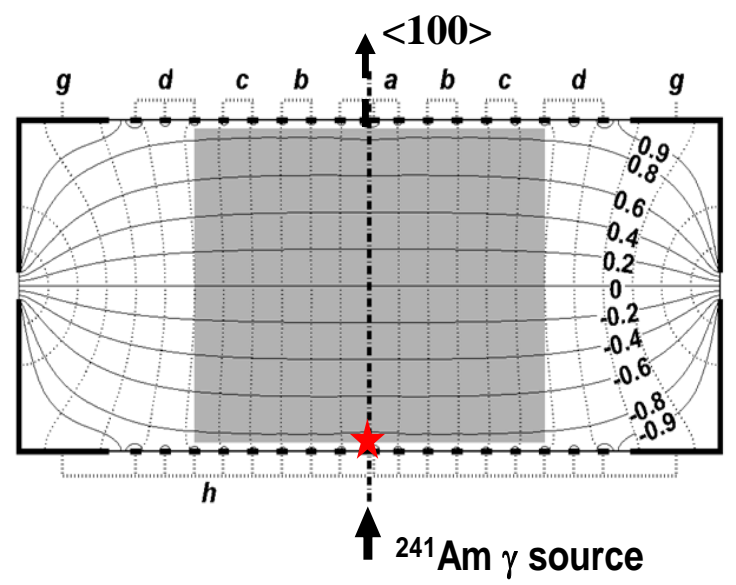

Fig. 1. Schematics of the detector in cross-section showing the connection scheme of the electrodes. The latter are represented as the thick lines (not to scale in the figure). The charge measurement channels are designated by $a$, $b, c, d, g$ and $h .^{2}$ Equipotentials and electric field lines (full and dotted lines) are drawn for top and bottom electrode biases $V_{p}=+1 \mathrm{~V}$ and $-V_{p}=-1 \mathrm{~V}$ relative to detector casing, respectively. The electric field is approximately uniform within the core of the crystal (shaded area). The red star marks the area of energy deposition for $60 \mathrm{keV}$ photons emitted by the ${ }^{241} \mathrm{Am} \gamma$ source. 


\section{Magnetic field effects on a cryogenic Ge detector for dark matter search}

a 200g coplanar grid detector of the Edelweiss collaboration (fig. 1). ${ }^{2}$ A collimated ${ }^{241} \mathrm{Am} \gamma$ source delivers $60 \mathrm{keV}$ photons, absorbed within a volume of a few $\mathrm{mm}^{3}$ just underneath the center of the bottom surface of the detector crystal. Comparative measurements on electrons and holes are thereby facilitated, as switching from one type of carrier to the other is simply done by inverting the bias polarities between the top and the bottom sets of electrodes. The detector is mounted in a copper casing attached to the mixing chamber of a ${ }^{3} \mathrm{He} /{ }^{4} \mathrm{He}$ dilution refrigerator. The entire setup (including the radiation shields and the ${ }^{4} \mathrm{He}$ dewar) is built from non-magnetic (copper and stainless steel) material. The dewar has a narrow section at the detector level, which permits to place an external magnet in a location relatively close $(\sim 10$ $\mathrm{cm}$ ) to the detector crystal. The magnet is mounted on a rotating arm, so that it can be removed to a distant position. Different field configurations are achieved depending on the orientation of the dipole axis of the magnet relative to the detector axis. The maximum field intensity at the location of the Ge crystal, as measured by a Hall probe, is $3.5 \mathrm{G}$ with the field parallel, and $7 \mathrm{G}$ with the field normal to the detector axis, respectively.

\section{EXPERIMENTAL RESULTS}

a) Cooling procedure. Cooling of the detector through the superconducting transition of aluminum $(1.2 \mathrm{~K})$ was performed in three different ways: without any applied magnetic field, and with the field of the magnet set parallel or normal to the detector axis. Whatever the method used, no difference was observed in subsequent measurements of electron and hole collection at $\mathrm{mK}$ temperatures, which indicates negligible flux trapping by the annular collection electrodes for these experimental conditions.

b) Magnetic field effects on electron and hole collection. Experiments reported elsewhere in these proceedings demonstrate large differences between electron and hole collection in Ge detectors at $\mathrm{mK}$ temperatures, with regard to carrier straggling especially. ${ }^{2-5}$ These differences are also reflected in the magnetic field effects (figs. 2 and 3). The largest effects are observed for holes, with the magnetic field normal to the detector axis (fig. 2 (a)). Hole drift in the absence of the magnetic field is along the $<100>$ detector axis, so that the carriers are collected by the $a$ set of electrodes. Application of the magnetic field entails a deflection of the carrier trajectories, so that a fraction of the holes are now collected by the $b$ electrodes. Scatter plots of the charge collection signals show a considerable dispersion from event to event in the amount of holes collected by the $a$ and $b$ channels (although their sum remains a constant, equal to the full collected charge as measured by the $h$ channel). A different situation obtains when the magnetic field is 


\section{A. Broniatowski}

Field normal to detector axis
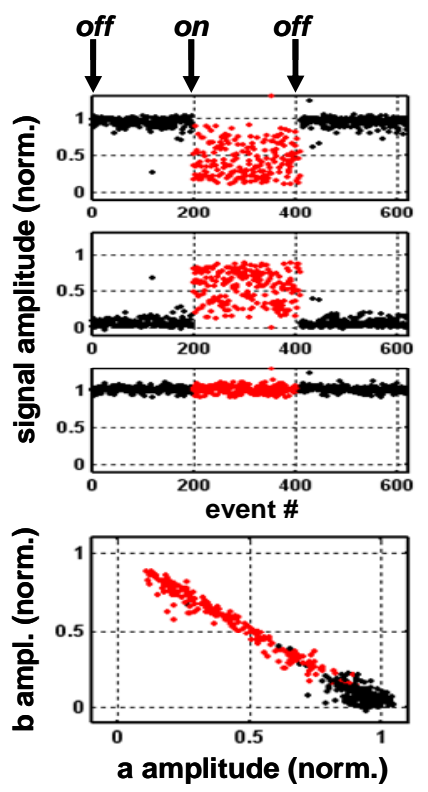

(a)
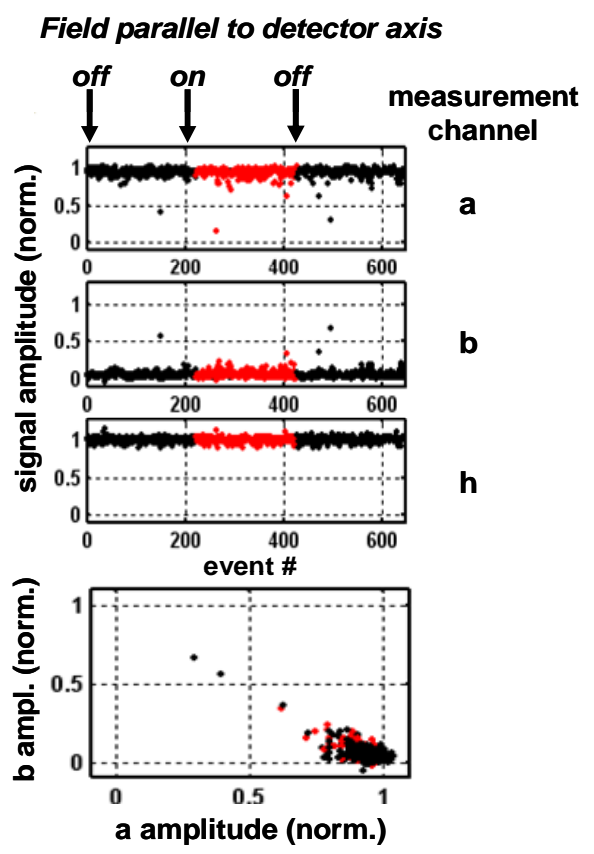

(b)

Fig. 2. (a): Scatter plots of hole collection signals in the $a, b$ and $h$ channels, with the dipole field of the magnet $(7 \mathrm{G})$ normal to the detector axis. The data in the other measurement channels are not represented, as they show zero signal amplitude. Detector bias $\mathrm{V}_{\mathrm{p}}=-0.5 \mathrm{~V} ; \mathrm{T}=20 \mathrm{mK}$. Amplitudes are normalized to unity for $60 \mathrm{keV}$ signals in the $h$ channel. The 'on' and 'off' labels refer to the location of the magnet in close and remote positions relative to the Ge crystal, with the corresponding (red and black) colors for the data points in the scatter plots. The graph at the bottom left shows correlation of the $a$ and $b$ signal amplitudes, whose values sum up to that in the $h$ channel. (b): same as (a), with the field (3.5 G) parallel to the detector axis (see text).

turned parallel to the detector axis, as the Laplace force on a carrier is now reduced to $\sim 0$, so that hole collection is hardly affected at all (fig. 2 (b)).

Compared with holes, electrons exhibit altogether different features of charge collection, due to the combined effects of effective mass anisotropy and the multivalleyed character of the conduction band. With the electric field along the $<100>$ direction, electrons in the different energy valleys propagate at an angle of $\sim 35$ degrees to the detector axis. Scattering processes, due to both phonons and impurities, lead to a statistical redistribution of the electrons among the different energy valleys as they are drifted to the 


\section{Magnetic field effects on a cryogenic Ge detector for dark matter search}

electrodes. There results a complex, bias dependent pattern of charge collection between the different measurement channels.,3 Application of a magnetic field leads to further changes in the collection patterns, as figure 3 (a) shows for the field in the direction normal to the detector axis. On the other hand, minute effects only are observed in the $c$ and $d$ channels when the field is turned parallel to the detector axis (fig. 3 (b)).

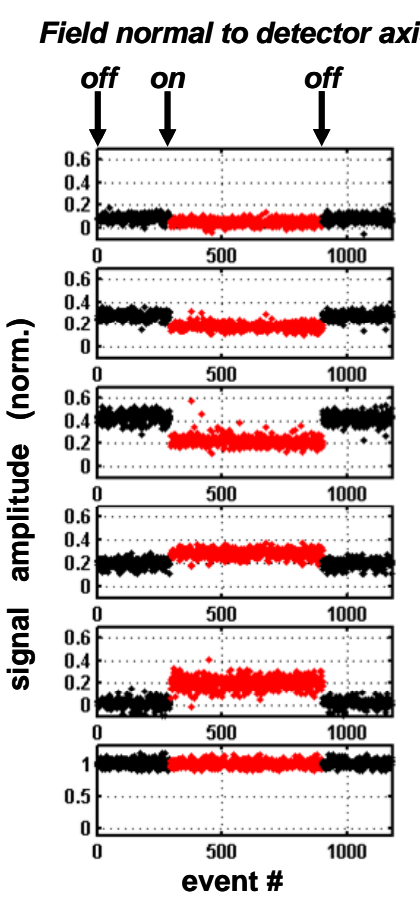

(a)

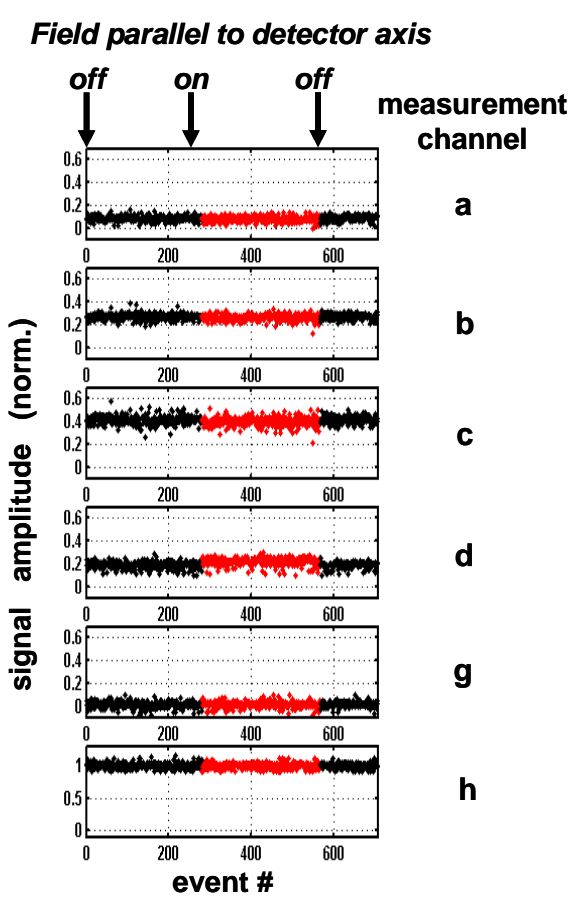

(b)

Fig. 3. (a): Scatter plots of electron collection signals in the $a, b, c, d, g$ and $h$ measurement channels, with the dipole field of the magnet $(7 \mathrm{G})$ normal to the detector axis. Detector bias $\mathrm{V}_{\mathrm{p}}=0.25 \mathrm{~V} ; \mathrm{T}=20 \mathrm{mK}$. (b): same as (a), with the field $(3.5 \mathrm{G})$ parallel to the detector axis.

c) Dependence of the magnetic field effects on the collection field. The data presented were obtained at detector biases $\left(\mathrm{V}_{\mathrm{p}}=0.25 \mathrm{~V}\right.$ for electrons and -0.5 $\mathrm{V}$ for holes), which correspond to a field strength in the bulk of the detector crystal of $\sim 0.22$ and $0.45 \mathrm{~V} / \mathrm{cm}$ respectively. As the field increases, the magnetic field effects on charge collection decrease in relative magnitude, until they become wholly undetectable for fields $>\sim 2 \mathrm{~V} / \mathrm{cm}$. 


\section{A. Broniatowski}

\section{DISCUSSION}

Although magnetic field effects on hot carrier transport in bulk Ge crystals were investigated as early as the 1960 's, ${ }^{6,7}$ we do not know of similar studies at $\mathrm{mK}$ temperatures. The present experiments are therefore of an exploratory nature. The electric field intensities in our measurements, small as they are (a fraction of a V/cm only), are commensurate with the collection field in actual dark matter detector operation. ${ }^{8}$ Although magnetic fields of a few gauss are unlikely within the cold stage of the dilution unit, such fields, if present, would affect electron and hole transport significantly.

The effects of a magnetic field on carrier transport are limited to the component of the field normal to the detector axis, whereas the parallel component shows little or no effect. Part of the explanation lays in the symmetry properties of electron and hole transport for the particular $(<100>)$ orientation of the electric field in our experimental setup. ${ }^{9,10}$ An additional factor is the superconducting character of the aluminum annular electrodes. Because the thickness of the electrodes $(200 \mathrm{~nm})$ is large compared with the London penetration depth $\left(\sim 50 \mathrm{~nm}\right.$ in $\mathrm{Al}$ at $\mathrm{mK}$ temperatures $\left.{ }^{11}\right)$, effects of flux conservation obtain through the electrodes, ${ }^{12}$ which also affect the geometry of the magnetic field within the bulk of the detector crystal. A local reinforcement of the field is expected in particular in the near-vicinity of the electrodes, which, if large enough, could bear upon charge collection for energy deposits in these areas of the detector. Because the voltage biases of the electrodes in actual detector operation ${ }^{8}$ differ from those achieved in the present set of measurements, the question arises then, whether a stray magnetic field of weak intensity (a few gauss typically) could also affect the ultimate background rejection capabilities of the detectors.

\section{REFERENCES}

1. J. Domange et al., J. Low Temp. Phys. (2012) Proceedings LTD14. doi: 10.1007/s10909-012-0535-5

2. E. Olivieri et al., J. Low Temp. Phys. (2012) Proceedings LTD14. doi: 10.1007/s10909-012-0548-0

3. A. Broniatowski, J. Low Temp. Phys. (2012) Proceedings LTD14. doi: 10.1007/s10909-012-0543-5

4. B. Cabrera et al., e-print arXiv:1004.1233v1 [astro-ph.IM] (2010).

5. V. Aubry-Fortuna and P. Dollfus, J. Appl. Phys. 108, 123706 (2010).

6. D. Chattopadhyay, Phys. Rev. B 7, 4739 (1973).

7. S. Komiyama and R. Spies, Phys. Rev. B 23, 6839 (1981).

8. A. Broniatowski et al., Phys. Lett. B 681, 305 (2009).

9. L. Reggiani et al., Phys. Rev. B 16, 2781 (1977). 
Magnetic field effects on a cryogenic Ge detector for dark matter search

10. C. Jacoboni et al., Phys. Rev. B 24, 1014 (1981).

11. J.F. Cochrant and D.E. Mapother, Phys. Rev. 111, 132 (1958).

12. F. London, Superfluids vol. 1 (Macroscopic theory of superconductivity), Wiley 1950. 